\title{
Post Spinal Fixation: What the surgeon wants to know
}
A Megahed Pranav Sharma, A Saeed Bamashmos, K Elfatairy, A Heshmatzadeh Behzadi, S Smith, Bridgeport Hospital, Yale New Haven Health

\begin{abstract}
There is an increase in number of spinal surgeries over the past decades. The main types of spinal surgeries are decompression, fixation, stabilization and fusion, correction of deformity, as well as lesion excision and debridement. Knowledge of the indications of the spinal surgery, the type and approach of procedure, the numerous spinal fixation techniques, the goals of the surgical interventions and normal and abnormal post-operative imaging findings is paramount to aid the radiologist in generating reports that adress the issue the surgeon wants to know to provide optimal patient management.
\end{abstract}

Aim of implanted hardware: Implanted hardware exists to provide shortterm stability while fusion develops.

Sequalae: Lost mobility of the fused segment places additional stresses on adjacent levels of the vertebrae. The consequence is an increased likelihood of degenerative changes, ligamentous instability, fracture at levels adjacent to successful fusion.

\section{Fusion Hardware:}

Screws and wires

Bone grafts and biologic materials Intervertebral disk prosthesis

Anterior screw

Allograft struts or bone graft cages

Static and extendable rods

Interbody cages

Pedicle screw and rod or plate

Autograft or allograft bone blocks
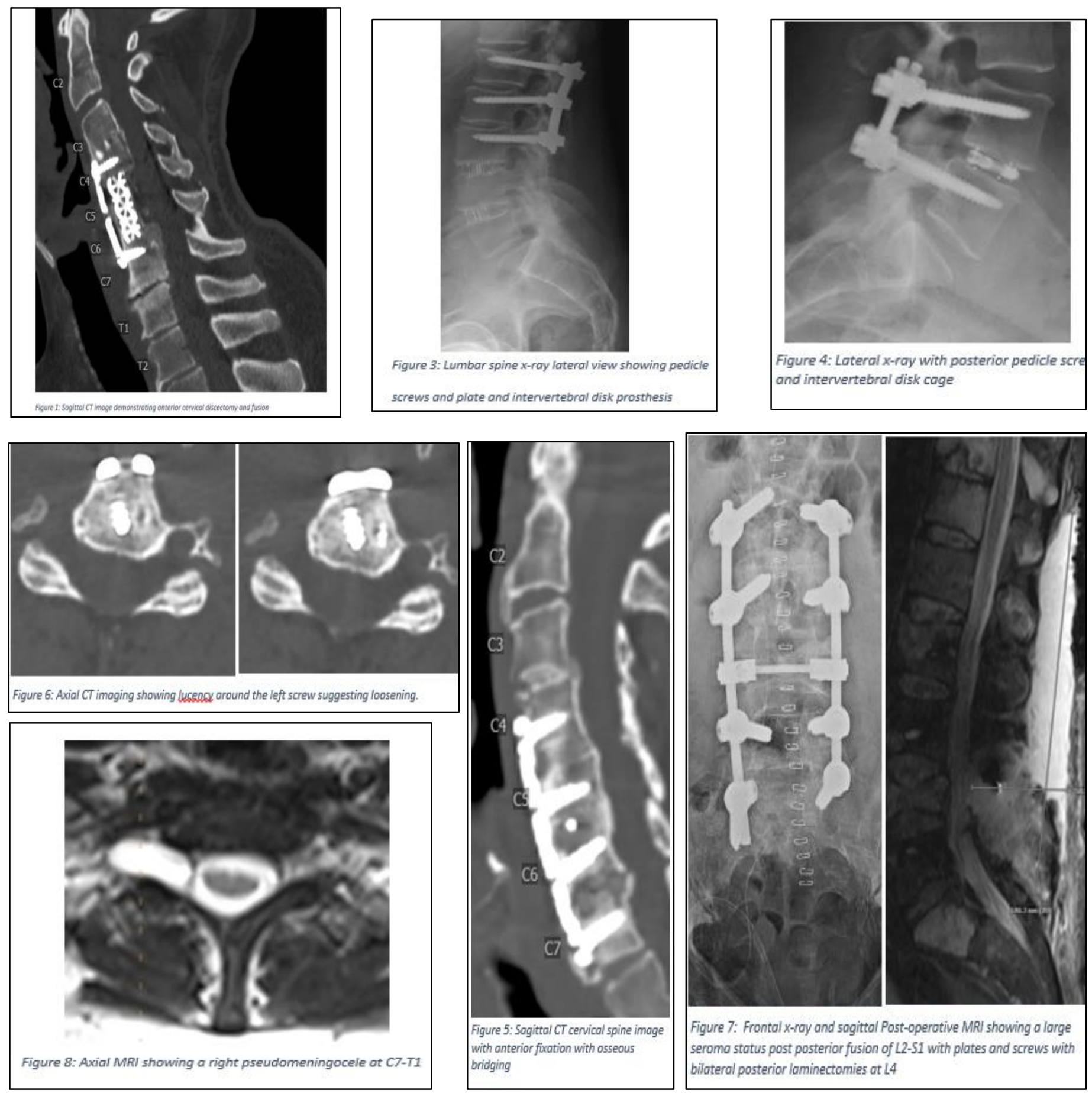

https://medapparatus.com/NeckSpine/Spine Page2.html

\section{Report:}

1. What is the surgery? What is the approach of hardware fixation

\section{a) Cervical:}

-Anterior cervical fusion plate

-Anterior cervical discectomy and fusion ( ACDF)

-Anterior cervical corpectomy and fusion

Anterior interbody fusion device

-Cervical disk replacement eg: PEEK disk cages

-Posterior cervical spinal instrumentation

-Anterior and posterior fusion

-Anterior and posterior fusion with intervertebral struts

b) Lumbar:

-Posterior lumbar interbody fusion

-Transforaminal lumbar interbody fusion

-Anterior lumbar interbody fusion

-Intervertebral disk spacers
2. Level of the surgery? (Compare to pre-surgery films)

3. Hardware material assessment/ Assess each element of the graft: Position, Alignment, Configuration, Graft material

-Transpedicular screws should traverse the central portion of the pedicle and enter the vertebral body parallel to the endplate without breaching the vertebral body cortex.

-Sacral fixation screws may penetrate the vertebral cortex for a short distance.

-Transpedicular C2 and C7 screws should have a horizontal orientation, extending anteriorly and slightly medially without breaching the pedicle cortex.

-Lateral mass screws in the C3-C7 vertebral bodies should have a superior and slightly lateral orientation.

-After ACDF, the anterior cervical plate should have a lordotic contour and should be flush against the vertebral bodies. There should be a distance of at least $5 \mathrm{~mm}$ between the superior and inferior margins of the plate and the adjacent endplates to prevent periplate ossification.

-An interbody graft is accurately positioned when the distance between the posterior graft margin and the posterior vertebral body margin is 2 $\mathrm{mm}$.

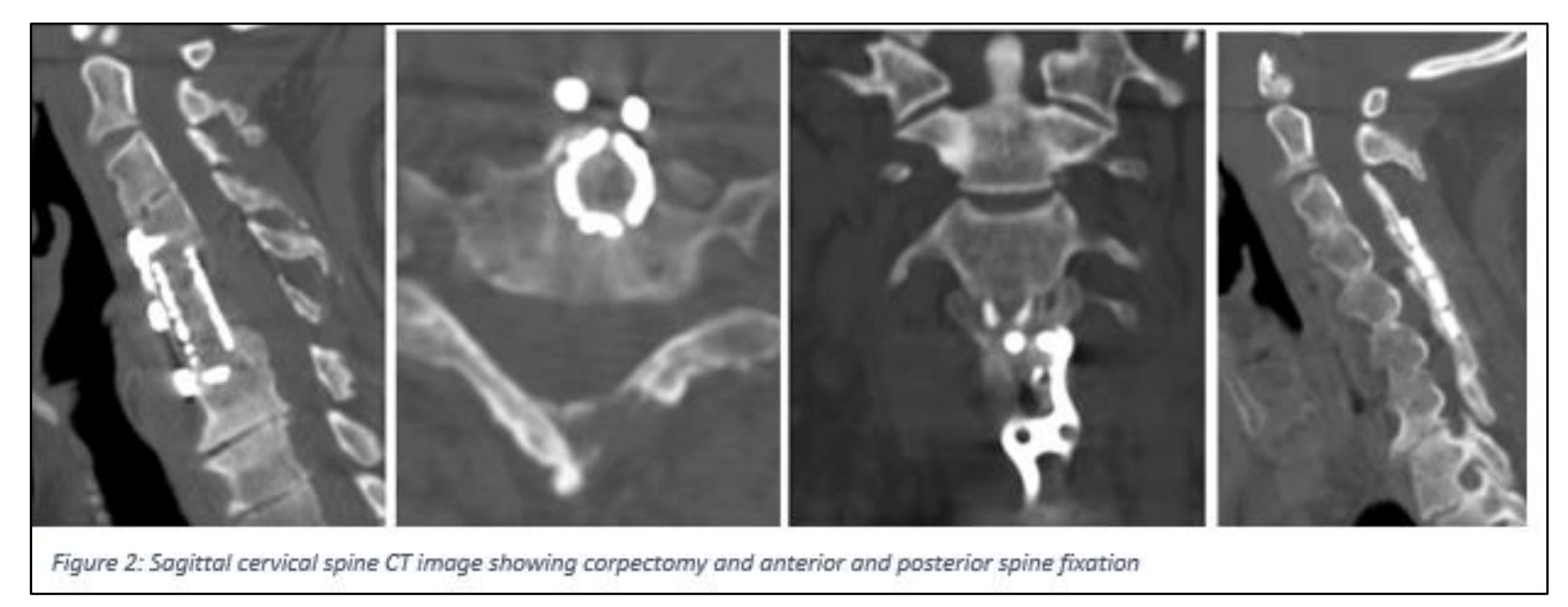

4. When was the surgery? What is the expected outcome for this time? Is it met?

*Signs of successful surgery*

-Signs of bridging bone should occur by 6-9 months after surgery

-One year after surgery, mature trabeculation and solid cortical bridging.

5. Normal post-operative findings:

Soft-tissue edema and small amounts of gas and fluid.

-Granulation or scar tissue along the surgical tract and peridural spaces.

-Paraspinal muscle atrophy with fat replacement.

6. Complications:

Hardware complications:

Angulation/Herniation

Fracture around implants

Loosening: Peri-implant osteolysis

Resorption of graft material

Failure of the graft material to incorporate into a solid fusion

Alignment complications:

Pseudoarthrosis

Fracture

Non-union

Complications of fusion

Adjacent level degenerative changes

Post-operative complications:

Hematoma, seroma, surgical site infection, abscess

Discitis, osteomyelitis, arachnoiditis, pseudomeningioceles, CSF fluid leaks

7 Assessment of alignment, fracture or dislocation

8. Assessment of the bony vertebrae

9. Assessment of the discs, neural foramina, thecal sac and central canal.

10. Assessment of the prevertebral soft tissues and adjacent structures. 